\title{
Enantioselectivity on Surfaces with Chiral Nanostructures
}

\author{
David M. Rampulla \\ Andrew J. Gellman \\ Carnegie Mellon University, Pittsburgh, Pennsylvania, U.S.A.
}

\section{INTRODUCTION}

Chirality is a critical property of amino acids that form proteins, the building blocks of life, and of DNA, which encodes the genetic traits passed from one generation to the next. Furthermore, chirality can be observed throughout nature in objects ranging from spiral seashells to the human hands. If an object and its mirror image are nonsuperimposable, then they have the property of chirality. This property is found in nearly every biological molecule and in many synthetic bioactive molecules such as pharmaceuticals and agrochemicals. From a practical perspective, the importance of molecular chirality arises from the fact that the two mirror images of a chiral molecule, known as enantiomers, can have vastly different physiological impacts when ingested by living organisms. As a result, it is necessary to produce many chiral compounds in enantiomerically pure form. ${ }^{[1]}$ Understanding chirality and enantioselectivity is critical to the development of the separations and reactions used to achieve enantiopurity.

Many processes used in chemical production involve solid surfaces and can be made enantioselective by using chiral surfaces. There are three common types of chiral surfaces: surfaces modified by chiral organic adsorbates, surfaces of any naturally chiral bulk crystalline solid, and chiral surfaces prepared from achiral bulk crystals. A number of studies have shown that such chiral surfaces exhibit enantiospecific properties when exposed to chiral species in either the gas phase or in solution. Although chirality is simply a symmetry property of an object or an extended lattice, the enantiospecificity of surfaces is derived from the local structure of nanoscale features such as molecular adsorbates, or specific arrangements of atoms at a crystal surface. Experimental and theoretical work to elucidate the properties of these nanostructured surfaces is an active area of research in surface chemistry. Although a number of aspects of the enantioselectivity of chiral surfaces are now understood, many unanswered questions remain as topics of ongoing research in the field.

\section{CHIRALITY OF MOLECULES, SOLIDS, AND SURFACES}

The simplest molecules exhibiting chirality are those containing a carbon atom tetrahedrally coordinated to four different substituents. Such molecules are not superimposable on their mirror images. Fig. 1 shows the tetrahedral arrangement of a simple, chiral carbon-centered molecule. The handedness of a chiral center or carbon atom is denoted $R$ for rectus (right-handed) or $S$ for sinister (left-handed) based on a convention conceived by Cahn, Ingold, and Prelog. ${ }^{[2]}$ Biologically relevant molecules can be very complex and tend to have multiple chiral centers. Although possession of one or more tetrahedral carbon atoms having four different substituents is sufficient to render a molecule chiral, it is not a necessary condition. Chiral centers can also exist in molecular structures such as allenes $\left(\mathrm{R}^{\prime} \mathrm{RC}=\mathrm{C}=\mathrm{CR}^{\prime} \mathrm{R}\right)$ and transcycloalkenes, which do not have tetrahedrally coordinated carbon atoms as their chiral centers.

Chirality can arise in crystalline solids because either the unit cell is chiral, or the basis of an achiral unit cell is chiral. Examples of the former case include all solids based on monoclinic unit cells. Examples of the latter case could be crystals of enantiomerically pure compounds. Of the 230 space groups into which all crystals can be classified, 65 are chiral. ${ }^{[3]}$ Because mirror planes, inversion centers, and glide planes in crystal structures render them achiral, the chiral space groups can only contain screw axes and rotational axes as symmetry elements.

Surfaces can have structures that are chiral in the sense that they are nonsuperimposable on their mirror images. One of the most common and versatile approaches to preparing a chiral surface is to adsorb an enantiomerically pure chiral compound onto the surface of an otherwise achiral substrate (Fig. 2a). The mere presence of such a chiral modifier or template renders the surface chiral. The second obvious class of chiral surfaces is those produced by cleaving any solid having a chiral bulk structure. Many oxides have chiral bulk structures and thus expose chiral surfaces (Fig. 2b). Although it is somewhat 


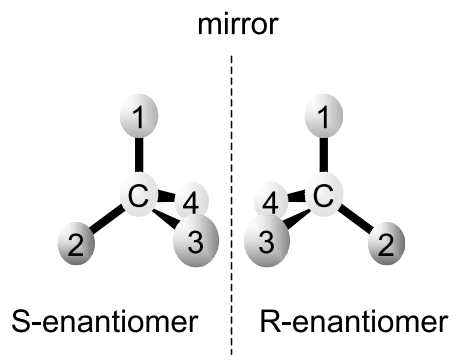

Fig. 1 The mirror images of chiral molecules are not superimposable on each other, thus simple molecules based on tetrahedrally coordinated carbon atoms with four different substituents are chiral. (View this art in color at www.dekker. com.)

counterintuitive, it is also possible to create chiral surfaces from crystalline solids with achiral bulk structures. Metals have either face-centered cubic (fcc), body-centered cubic (bcc), or hexagonal close-packed (hcp) bulk structures, all of which are achiral. Nonetheless, many of their high Miller index surfaces have monoclinic lattices and thus are chiral. The fcc(643) surface shown in Fig. 2c is composed of terrace-step-kink features, which render it chiral. On all three types of chiral surfaces, the features that give rise to chirality have nanometer dimensions. This nanostructure is critical to their utility in enantioselective chemistry. In order for adsorbate-surface interactions to be enantiospecific, the length scale of the chiral structures on the surface must roughly match the length scale of the chiral features of the adsorbed molecule.

\section{IMPORTANCE OF CHIRAL SURFACES}

The two enantiomers of a chiral compound are structurally related simply by mirror symmetry and, thus, enantiomerically pure compounds have bulk properties such as density, melting point, heat of phase change, etc. that are truly identical. The properties of two enantiomers are only differentiated in a chiral environment and, in particular, in a chiral environment in which the length scale of the chiral features is on the nanoscale matching molecular dimensions. Living organisms constitute chiral environments because the proteins that perform most biochemical functions in living organisms are chiral and exist as single enantiomers. As a result, the physiological impact of the two enantiomers of a chiral compound will be different. Appreciation of this fact could have prevented one of the greatest tragedies in medical history. In the late 1950s, the chiral drug thalidomide was administered as a racemic mixture (equimolar concentrations of both enantiomers) to pregnant women to abate morning sickness and to act as a general sedative. Unfortunately, only the $R$ - enantiomer has the desired therapeutic effect whereas the $S$-enantiomer is a teratogen and caused birth defects in thousands of infants born in that period of time. ${ }^{[4]}$ As a consequence of this problem and related issues associated with the use of many chiral pharmaceuticals, the production of enantiomerically pure drugs has grown to be a large concern for the pharmaceutical industry. The current market for enantiomerically pure pharmaceuticals is estimated to be in excess of US\$100 billion per year. ${ }^{[5]}$

As a result of the fact that the physical properties of the two enantiomers of chiral compounds are identical, most chemical syntheses lead to the production of equimolar or racemic mixtures of both enantiomers. If one desires an enantiomerically pure product, the racemic mixture must be separated. The separation of mixed products of a chemical synthesis is commonplace and can be achieved by a number of means; however, the separation of racemic mixtures is very difficult. The most common separation methods exploit differences in physical properties such as boiling point, melting point, or density; however, in achiral environments, these properties are identical for two enantiomers. Separation of racemic mixtures must be performed in chiral environments that differentiate the properties of the two enantiomers. For example, mixing two enantiomers $R_{1}$ and $S_{1}$ with a single enantiomer of a second chiral compound $\left(R_{2}\right)$ can result in the formation of diastereomeric complexes $R_{1} R_{2}$ and $S_{1} R_{2}$ (molecules with more than one chiral center that are not mirror images). These complexes are not enantiomers of one another and thus can be separated by exploiting differences in their physical properties. ${ }^{[6]}$ Once the two complexes are separated, $R_{2}$ can be removed and separated to produce pure samples of $R_{1}$ and $S_{1}$. The desired enantiomer can then be used, whereas the

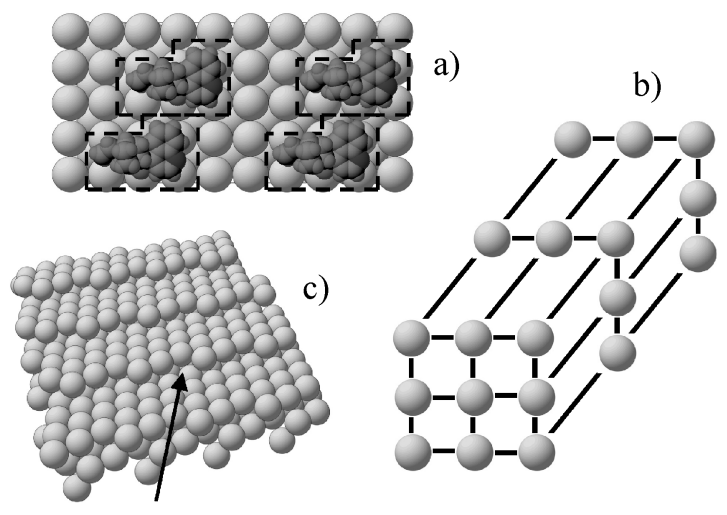

Fig. 2 Chiral surfaces: (a) modification of an achiral surface with chiral organic molecules renders the surface chiral; (b) cleavage of bulk chiral solids exposes surfaces with chiral structures; and (c) cleavage of an achiral fcc metal along a high Miller index plane generates a surface with chiral kinks. (View this art in color at www.dekker.com.) 
undesired enantiomer is either discarded or, if possible, racemized and subjected to further enantioselective purification by complexation with $R_{2}{ }^{[6]}$ The point is that the addition of the pure enantiomer $R_{2}$ to the racemic mixture constitutes the creation of a chiral environment in which $R_{1}$ and $S_{1}$ can be separated.

Most enantiomerically pure substances are formed by homogeneous syntheses and then separated using techniques such as the aforementioned creation of diastereomers. Direct enantiospecific syntheses or simpler separation methods would, of course, be preferable. Many such processes, including heterogeneous catalyses and chromatographic separations, are commonly performed using solid surfaces. The types of chiral surfaces that are the subject of this entry have many potential applications in enantiospecific chemical processing.

\section{TYPES OF CHIRAL SURFACES}

Three types of nanostructured chiral surfaces will be described in this entry: those produced by chiral templating with an organic ligand, surfaces derived from chiral bulk structures, and those produced from achiral bulk structures such as metals. In all three cases, the length scale of the chiral features is that of nanometers and thus they can interact enantiospecifically with chiral adsorbates.

\section{Surfaces Templated with Chiral Organic Modifiers}

The adsorption of enantiomerically pure chiral molecules on achiral surfaces yields surfaces that are chiral, provided that the adsorbed molecule retains its chirality. Such surfaces can be used for enantioselective heterogeneous catalysis ${ }^{[7,8]}$ or enantioselective chromatography. ${ }^{[9,10]}$ The most successful chirally templated, enantioselective heterogeneous catalyst has been $\mathrm{Ni}$ templated with tartaric acid, which is used for the asymmetric hydrogenation of $\beta$-ketoesters and Pt templated with cinchonidine used for hydrogenation of $\alpha$-ketoesters (see Fig. 3). Many of the underlying issues encountered with these catalysts are common to other template/substrate systems, so this section will focus on these two examples.

\section{Hydrogenation of $\beta$-ketoesters on nickel}

The enantioselective hydrogenation of $\beta$-ketoesters on chirally templated Raney Ni catalysts has been reviewed extensively by Izumi. ${ }^{[11]}$ Most works have used Raney Ni and nickel powder as catalysts; however, some have used $\mathrm{Ni}$ particles supported on high-surface-area materials such as silica and alumina. ${ }^{[12]}$ These catalysts have demonstrated enantioselectivity for the hydrogenation of methyl acetoacetate to methyl-3-hydroxybutyrate, yielding enantiomeric excesses as high as $e e=95 \%$. ${ }^{[13]}$

The enantioselectivity of the catalytic hydrogenation of $\beta$-ketoesters is influenced by the nature of the $\mathrm{Ni}$ used as the catalyst, by the nature of the chiral modifier, and, in some cases, by the nature of a comodifier. In addition to tartaric acid, which has been shown to be the most promising modifier, $\alpha$-amino acids have been used. The superiority of tartaric acid is believed to arise from its ability to stereospecifically orient the reacting $\beta$-ketoesters on the surface via hydrogen bonding. ${ }^{[14]}$ One model has been suggested to explain the enantioselectivity in which chiral five-member chelate rings are formed by the bonding of the tartaric acid to Ni through a carboxylate oxygen atom and the oxygen atom of the $\beta$-hydroxyl group. ${ }^{[15]}$ a)

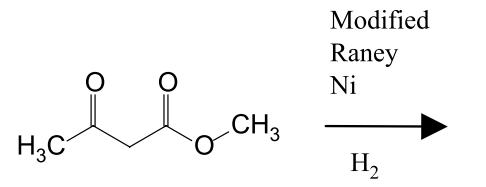

methyl acetoacetate

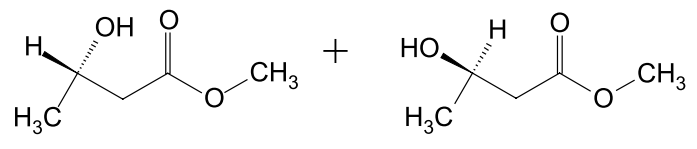

methyl-3-hydroxybutrate b)

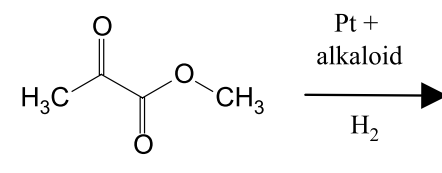

methyl pyruvate

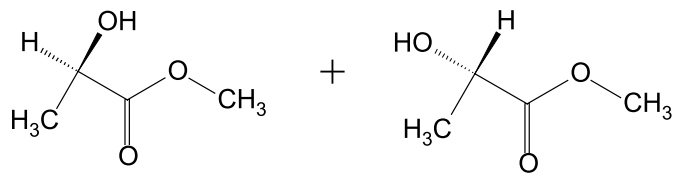

methyl lactate

Fig. 3 The asymmetric hydrogenations of methyl acetoacetate on $\mathrm{Ni}$ (a) and methyl pyruvate on Pt (b) are the two most widely studied enantioselective reactions catalyzed by chirally templated surfaces. 
In addition to the nature of the chiral modifier, another factor that determines the enantioselectivity of Ni-based catalysts is the size of the $\mathrm{Ni}$ particles. During the modification process of the Ni particles, tartaric acid has been shown to selectively dissolve catalyst particles with diameters less than $2 \mathrm{~nm}$. The tartaric acid creates the pores that contain the hydrogenation sites, and the longer a particle is leached, the more pores it will have. This supports earlier results showing that larger nickel particles yield higher enantioselectivity. ${ }^{[15-17]}$

Comodifiers can also influence the enantioselectivity of chirally templated surfaces. Typically, $\mathrm{NaBr}$ is used as the comodifier and is thought to poison the racemic sites, reaction sites that have no enantioselective bias for hydrogenation, thus increasing the net enantiomeric excess. ${ }^{[18]}$ However, it has also been proposed that the $\mathrm{NaBr}$ modifies the stereochemistry of the product-determining surface complex between the reactant and the chiral template. ${ }^{[19]}$

\section{Hydrogenation of $\alpha$-ketoesters on platinum}

The most highly studied enantioselective reaction over a chirally modified heterogeneous catalyst is the hydrogenation of $\alpha$-ketoesters over Pt templated with cinchonidine. Studies of this reaction began in 1978 when Orito et al. ${ }^{[20]}$ enantioselectively hydrogenated methyl pyruvate to $R$-(+)-methyl lactate in the presence of adsorbed cinchonidine. As in the case of the enantioselective hydrogenation on modified $\mathrm{Ni}$ catalysts, a variety of factors influence the enantioselectivity, including: temperature, the nature of the solvent, modifier concentration, and the size of the Pt particles.

One important aspect of the chirality of the cinchonatemplated $\mathrm{Pt}$ surface is the structure and adsorption geometry of the cinchona alkaloid ${ }^{[21]}$ illustrated in Figs. 4 and 5. There are three available sites for reaction. The first is the chiral cavity where the methyl pyruvate is bound to two or three cinchonidine molecules in an ordered array, the second is the 1:1 interaction between methyl pyruvate and a single cinchonidine molecule, and the third is a racemic site. One important characteristic of cinchonidine is its L-shaped configuration. Studies have shown that replacing the modifier with a chemically similar alkaloid that is not L-shaped, while increasing the reaction rate, eliminates enantioselectivity. ${ }^{[22]}$

Despite the large number of factors influencing the enantioselectivity of templated $\mathrm{Pt}$ catalysts, excellent results have been achieved by empirical tuning of $\mathrm{pH}$, solvents, particle sizes, and comodifiers as was done with the $\mathrm{Ni} /$ tartaric acid system. Blaser et al. ${ }^{[23]}$ obtained an $e e=94 \%$ for the hydrogenation of methyl pyruvate. As in the case of the hydrogenation of $\beta$-ketoesters on $\mathrm{Ni}$, the exact impact of these parameters must ultimately

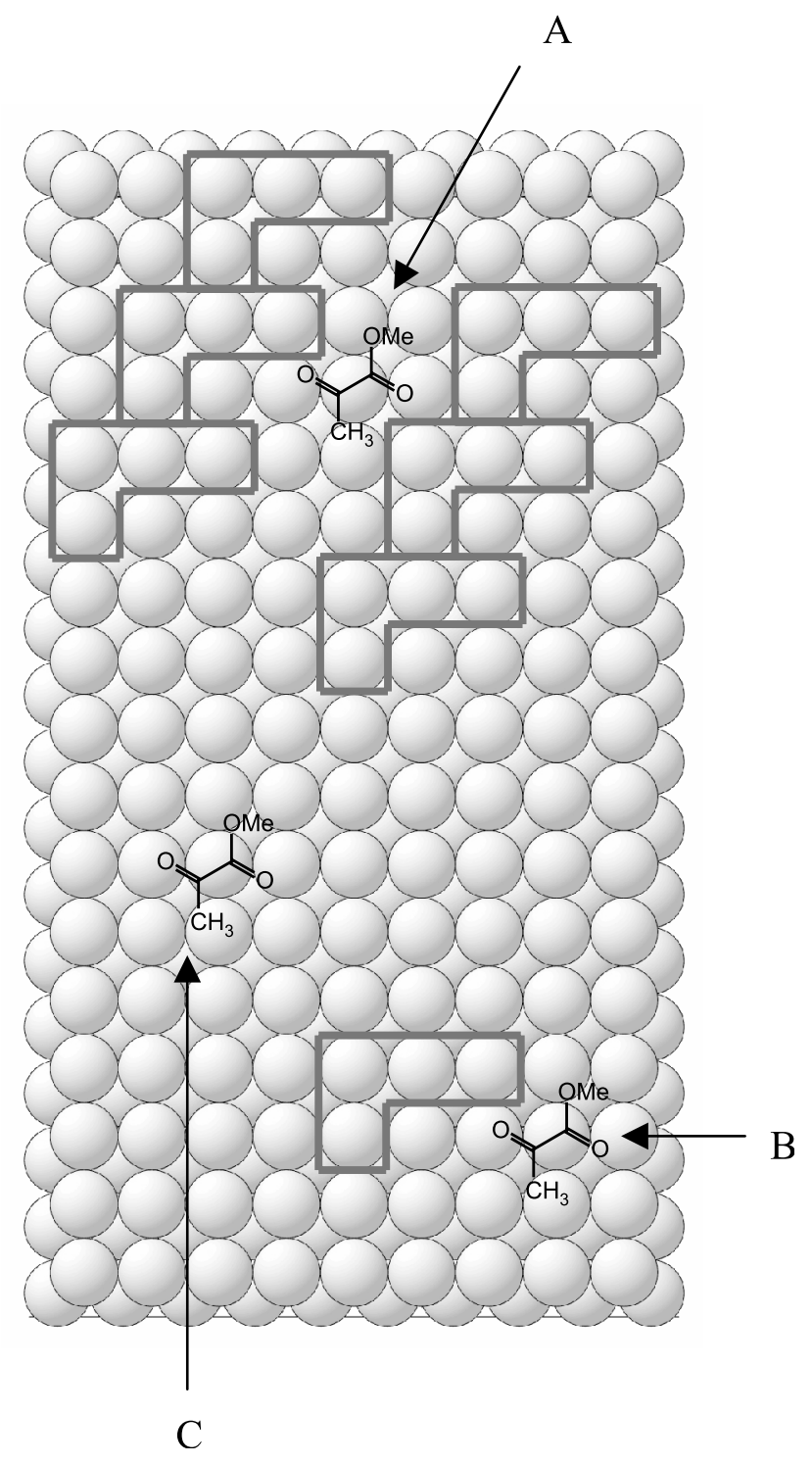

Fig. 4 It has been proposed that the L-shaped structure of the cinchona alkaloid creates three possible reaction sites when adsorbed on a surface. Sites A and B allow the methyl pyruvate to interact with the adsorbed modifier and thus promote enantioselective hydrogenation. Site $\mathrm{C}$ is achiral and does not promote enantioselective hydrogenation. (View this art in color at www.dekker.com.)

be elucidated to attain enantioselectivities approaching $100 \%$.

\section{Other chiral templates}

Three structural features of the cinchona modifier are thought to impart chirality to the Pt surface. ${ }^{[7]}$ First, $\pi$ bonding through the quinoline ring system is believed to anchor the modifier to the surface; second, the chirality of 


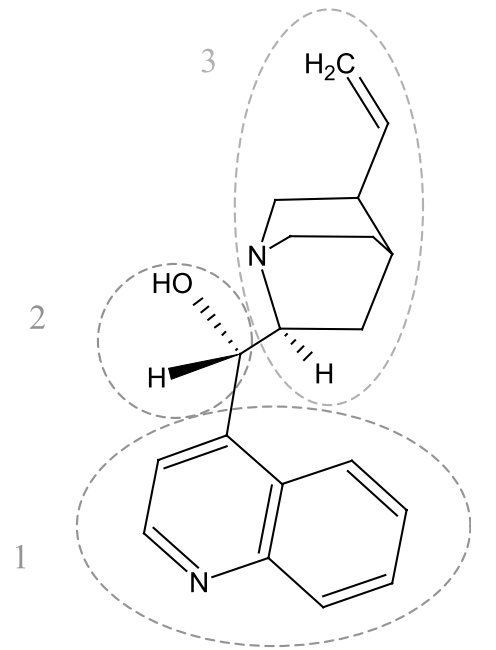

Fig. 5 It has been suggested that three features of the cinchonidine structure are necessary to impart enantioselectivity. First, the quinoline ring system (1) is believed to anchor the modifier to the surface; second, the chirality of the reaction product is directed by the chiral region between the quinoline and the quinulcidine rings (2); and, third, the tertiary nitrogen in the quinulcidine ring (3) interacts with the $\mathrm{C}=\mathrm{O}$ in the reacting ketoester. (View this art in color at www.dekker.com.)

the product is directed by the chiral region between the quinoline and quinuclidine rings; and, third, the tertiary nitrogen in the quinuclidine ring interacts with the $\mathrm{C}=\mathrm{O}$ in the ketoester (Fig. 5). Based on this model for the origin of the enantioselectivity of catalysts templated with cinchonidine, new modifiers based on the cinchona alkaloid, such as dihydroapovincaminic acid ethyl ester, ${ }^{[24]}$ strychnine, ${ }^{[25]}$ and others, ${ }^{[26,27]}$ have been identified and have been shown to work with varying degrees of success.

Amino acids have also been identified as potentially useful chiral modifiers for catalytic surfaces. ${ }^{[25]}$ In particular, the adsorption of glycine ${ }^{[28-30]}$ and alanine ${ }^{[31,32]}$ has been studied on $\mathrm{Cu}(110)$. These amino acids form well-ordered arrays whose structure is driven by hydrogen bonding. Alanine has been shown to be a promising modifier on $\mathrm{Cu}(110)$ because of the existence of a homochiral, two-dimensional phase that extends across the surface. ${ }^{[32]}$

\section{Surfaces Based on Bulk Chiral Materials}

In addition to chiral surfaces that have been templated with chiral ligands, naturally chiral surfaces can be derived from inherently chiral bulk crystalline structures. This can be performed either by exposing a surface of a crystal of an enantiomerically pure organic compound, or by exposing a surface of a naturally chiral crystal. It has been postulated that the chirality of certain naturally occurring minerals may have been critical to the homochiral evolution of life on Earth. The homochirality of naturally occurring biomolecules (i.e., the preference for L-amino acids and D-sugars) may have arisen from enantioselective adsorption on naturally chiral crystal surfaces. ${ }^{[33]}$

\section{Surfaces of bulk enantiomerically pure compounds}

The crystallization of an enantiomerically pure sample of an organic compound will produce a crystal structure that must be chiral. Similarly, the surfaces of such a crystal must be chiral and should have enantiospecific properties. This ought to offer an excellent avenue for the study of enantiospecific interactions between chiral molecules; however, there do not seem to have been any significant attempts to study the surface chemistry of such materials.

\section{Surfaces of bulk enantiomorphic crystals}

Intrinsically chiral surfaces can be produced from crystalline materials with inherently chiral bulk structures. One of the most common chiral materials is quartz, whose bulk structure is formed by a helical arrangement of corner-sharing $\mathrm{SiO}_{4}$ tetrahedra. This helical structure is chiral and renders quartz chiral. There have been some studies of the enantioselectivity of the surfaces of quartz crystals. However, much of this work has used powdered samples, which expose a variety of different crystallographic planes, each of which will have different adsorption characteristics for the same chiral species. ${ }^{[34,35]}$ Bonner et al. ${ }^{[36,37]}$ studied enantioselectivity on quartz powders prepared from enantiomerically pure crystals. By comparing the adsorbed concentrations of Dalanine and L-alanine on the same faces of quartz, a preferential enantioselective adsorption of approximately $1.4 \%$ was observed for D-alanine on right-handed quartz and for L-alanine on left-handed quartz. In another demonstration of enantiospecific surface chemistry, synthesis of pyramidal alkanols with an enantiomeric excess of $e e>90 \%$ was initiated using quartz and sodium chlorate crystals. $^{[38]}$

Obviously, any material with a bulk chiral structure can expose chiral enantioselective surfaces; however, even achiral minerals can expose chiral surfaces. In an achiral environment, these would have equal surface energies and would be present in equal areas. However, it is possible to prepare achiral minerals, which expose surfaces with net chiral excess when grown in the presence of chiral molecules. When grown in the presence of chiral organic compounds, gypsum displayed an asymmetric growth. ${ }^{[39]}$ Calcite, another achiral crystal, has also displayed asymmetric growth in the presence of chiral amino acids. ${ }^{[40]}$ 


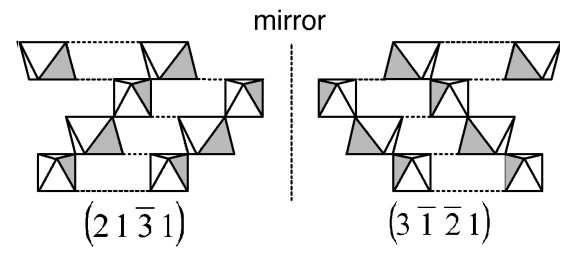

Fig. 6 On certain faces of calcite, the $\mathrm{CaO}_{6}$ octahedra are arranged in a chiral structure. (View this art in color at www.dekker.com.)

The origin of homochirality in living systems is one of the most intriguing aspects of the development of life on Earth. It has been proposed that the enantioselective processes that must have led to this homochirality occurred on chiral surfaces. To study the origin of the homochirality of life on Earth, Hazen et al. ${ }^{[41]}$ have chosen to use calcite, an achiral mineral, which can expose chiral surfaces. Fig. 6 shows the chiral facets of crystalline calcite. This mineral was abundant during the Archaean Era, a time in the Earth's history when the homochirality of life is thought to have originated. Furthermore, amino acids have particularly high heats of adsorption on calcite.

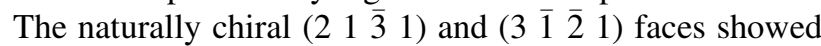
$10 \%$ enantioselective adsorption of D-aspartic acid and Laspartic acid, respectively. A control experiment using the achiral $\left(\begin{array}{llll}1 & 0 & 1 & 1\end{array}\right)$ face displayed no preference for aspartic acid adsorption.

Most of the known crystalline materials with naturally chiral bulk structures are covalent inorganic solids such as oxides, which have surfaces with low chemical reactivity. One approach to enhancing their reactivity while maintaining enantioselectivity is through deposition of a transition metal film on their surfaces. Terent'ev et al. ${ }^{[42]}$ and Klabunovskii and Patrikeev ${ }^{[43]}$ created a nickel-coated quartz catalyst that enantioselectively hydrogenated $\alpha$ phenyl cinnamic acid. The mechanism by which the chiral substrate imparts enantioselectivity to the metal is not clear. On one hand, it may induce chirality in the structure of the metal film. Alternatively, the quartz might orient the reactant enantiospecifically at the edges of $\mathrm{Ni}$ particles where they may be hydrogenated. In principle, metal deposition onto chiral substrates is widely applicable to chiral surfaces with low reactivity; however, few works have been done in this field.

\section{Chiral Surfaces from Achiral Bulk Crystal Structures}

Although it may seem counterintuitive, chiral surfaces can be formed from crystalline materials with otherwise achiral bulk structures. This type of chiral surface is created by exposing a high Miller index plane with indices (hkl) such that $h \neq k \neq l$ and $h \cdot k \cdot l \neq 0$. Most of such surfaces have structures formed of terraces, steps, and kinks, as shown in Fig. 2c. It is the kinks on such surfaces that impart chirality. Each kink is formed by the intersection of three low Miller index microfacets $(\{111\},\{100\}$, and \{110\}) with different structures. ${ }^{[44]}$ The chirality of the kink arises from the sense of rotational progression among the three microfacets when viewed from above the surface. Like chiral molecules, the handedness of chiral surfaces can be denoted by using a modified Cahn-Ingold-Prelog convention based on microfacet priority. ${ }^{[4]}$ For fcc metals, the order of priority is $\{111\}>\{100\}>$ $\{110\}$ and surfaces with a counterclockwise progression of microfacets when viewed from above are denoted $(h k l)^{\mathrm{S}}$, whereas surfaces with a clockwise progression are denoted $(h k l)^{\mathrm{R}}$. The naming convention and microfacet structure are illustrated in Fig. 7.

If one considers the high Miller index surfaces with terrace-step-kink structures and recognizes that these are formed by the intersection of three different low Miller index microfacets, it is clear that there are six types of chiral terrace-step-kink structures that can be formed on the surfaces of achiral bulk structures. The full set of surfaces that can be derived from an fcc bulk structure is usually represented by the stereographic projection shown in Fig. 8. The points on the perimeter of the stereographic projection represent surfaces that are achiral. The six types of chiral kinks divide the interior of the stereographic projection into six regions. The differences in the structures of the surfaces within each region are the lengths of the steps that separate the kinks and the widths of the terraces that separate the steps. The points along the dashed lines of Fig. 8 that separate the regions with different types of kinks represent surfaces with structures that have kinks formed of single unit cells of the low Miller index microfacets. They are also chiral but cannot be thought of as having structures with long step edges separating the kinks. Finally, at the center of the stereographic projection is the (531) surface with a structure that is formed of single unit cells of the (111),

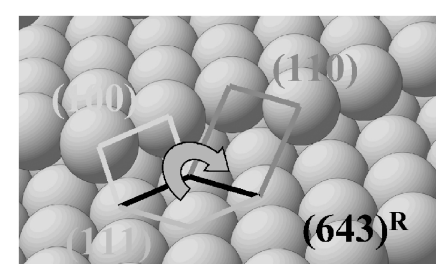

Fig. 7 A kink on a high Miller index surface of an fcc structure is formed by the intersection of three dissimilar low Miller index microfacets $(\{111\},\{100\}$, and $\{110\})$. Its handedness is dictated by the direction of rotational progression of the microfacets about the surface normal. (View this art in color at www.dekker.com) 


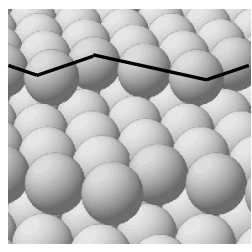

(653)

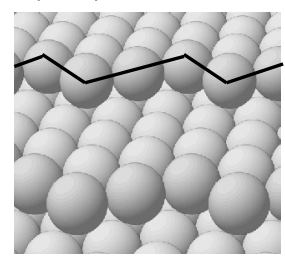

(111)

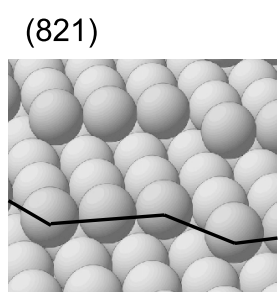

(100)

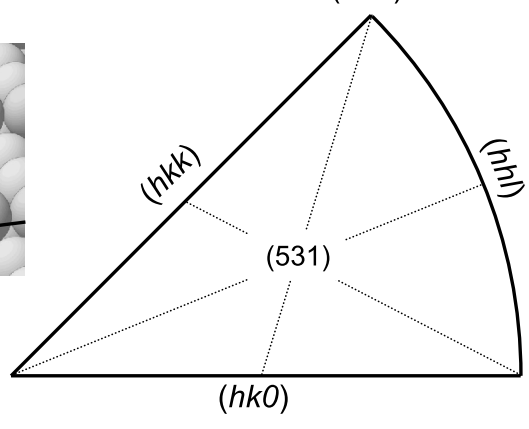

$(17,5,1)$

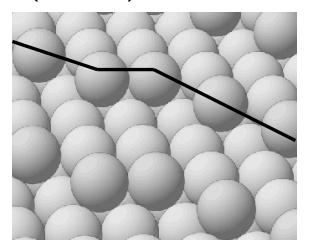

$(13,9,1)$

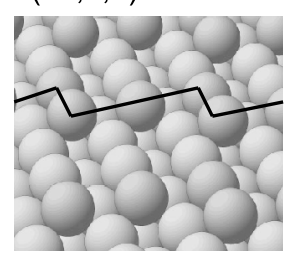

Fig. 8 The stereographic projection allows a representation of all possible surfaces exposed by cleavage of an fcc structure. The points on the perimeter of the triangle represent achiral surfaces. The points contained within the triangle represent surfaces with kinked structures that are chiral. The dashed lines divide the triangle into six regions containing surfaces with six different kink structures represented by the examples shown in the ball diagrams. At the center of the triangle is the (531) surface, which is comprised of single unit cells of the (111), (100), and (110) microfacets and has the highest kink density of all the chiral surfaces. (View this art in color at www.dekker.com.)

(100), and (110) microfacets. It is also chiral but cannot be thought of as having a terrace-step-kink structure. In summary, the high Miller index surfaces of cubic lattices have structures that expose chiral kinks of a small number

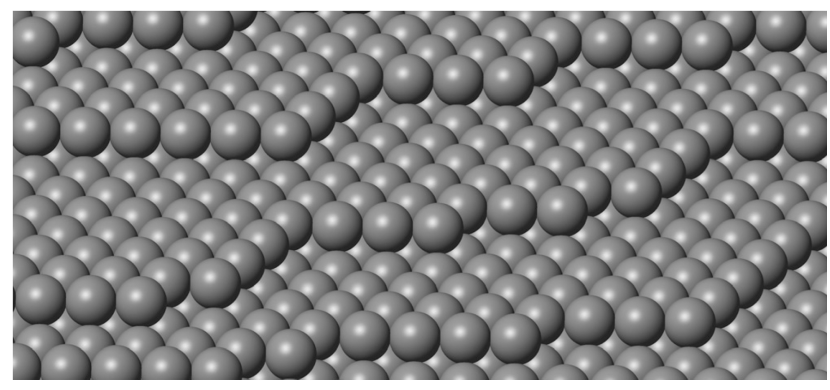

Fig. 9 A thermally roughened surface no longer exhibits the periodic kink structure seen in Fig. 2c. Because of kink coalescence, a thermally roughened surface has fewer kinks than an ideal surface, and these kinks are now formed by the intersection of longer step edges. Despite roughening, the surface maintains its chirality. (View this art in color at www.dekker.com.) of types. These chiral kinks are based on the nanoscale arrangements of atoms at the intersections of microfacets.

\section{Roughening of naturally chiral surfaces}

The ideal chiral surface structures that one obtains by simple cleavage of a fcc lattice along an high Miller index plane are formed of terrace-step-kink structures with nanoscale dimensions. The chiral kinks on the ideal surfaces are always single atom kinks along the step edge. These ideal structures will be destroyed by any amount of atomic motion or diffusion across the surface. A real chiral surface deviates from the ideal surface because of thermal roughening as seen in Fig. 9. Simulations by Sholl et al. ${ }^{[45]}$ Asthagiri et al. ${ }^{[46]}$ and Power et al. ${ }^{[47]}$ show that chiral surfaces roughen to form structures with nonideal kinks formed by the intersections of long step edges. Scanning tunneling microscopy of the $\mathrm{Cu}(5,8,90)$ surface has revealed such roughening of the step edges because of thermal diffusion of the kinks. ${ }^{[48]}$ However, it is important to realize that the nonideal kinks generated by step 
roughening are still chiral because they are formed by the intersections of three different low Miller index microfacets. Equally important, the net chirality of the surface is preserved because the kinks present on the roughened surface are predominantly of the same chirality as the kinks on the ideal high Miller index surface from which it evolved.

\section{Enantioselectivity of naturally chiral metal surfaces}

McFadden et al. ${ }^{[49]}$ first postulated that the naturally chiral, high Miller index planes of fcc metals ought to exhibit enantiospecific surface chemistry. Atomistic simulations by Sholl ${ }^{[50]}$ of the interactions of small chiral hydrocarbons with chiral platinum surfaces demonstrated that such enantioselectivity should occur and that enantiospecific adsorption energies for chiral molecules on chiral surfaces should be observable. Recently, temperatureprogrammed desorption studies of $R$-propylene oxide and $S$-propylene oxide ${ }^{[51,52]}$ and $R$-3-methyl-cyclohexanone ${ }^{[52,53]}$ on the $\mathrm{Cu}(643)^{\mathrm{R}}$ and $\mathrm{Cu}(643)^{\mathrm{S}}$ surfaces have revealed enantiospecific adsorption energies. The enantiospecific differences in the adsorption energies of propylene oxide and 3-methyl-cyclohexanone on the $\mathrm{Cu}(643)$ surfaces are $\Delta \Delta E_{\mathrm{ads}}=0.06 \mathrm{kcal} / \mathrm{mol}$ and $\Delta \Delta E_{\mathrm{ads}}=0.22$ $\mathrm{kcal} / \mathrm{mol}$, respectively. It is these types of differences in energetics that ultimately lead to enantioselective separations and enantioselective catalytic reactions on chiral surfaces. Such an enantioselective separation of racemic 3-methyl-cyclohexanone has been demonstrated on the $\mathrm{Cu}(643)$ surface. ${ }^{[53]}$

In addition to influencing the energetics of adsorption and desorption, the handedness of naturally chiral surfaces can influence the kinetics of surface reactions. Cyclic voltammetry has been used to study the electrooxidation kinetics of glucose and other sugars on an array of naturally chiral platinum electrode surfaces in aqueous solutions. ${ }^{[4,54-56]}$ These studies have shown that the rates of D-glucose and L-glucose oxidation on naturally chiral Pt electrodes can differ by as much as a factor of three. As in the case of enantioselective hydrogenation on the chirally templated Pt catalysts, the enantioselectivities of glucose oxidation are influenced by the presence of modifiers adsorbed from solutions. In particular, the enantioselectivity of glucose oxidation increases in the presence of sulfuric acid, but decreases in the presence of perchloric acid.

\section{Enantiomorphic heteroepitaxial growth of metals on chiral oxides}

The nanoscale kinks present on the high Miller index metal surfaces have clearly been shown to exhibit enan- tiospecific surface chemistry. However, the ultimate utility of surfaces with such chiral structures is dependent on their being produced with high surface area. One approach is to use the chiral surfaces of relatively cheap metal oxides as the substrates for deposition of otherwise expensive catalytic metals such as $\mathrm{Pt} .{ }^{[57]}$ This is analogous to the enantioselective catalyst mentioned above that was created by the deposition of $\mathrm{Ni}$ onto quartz. ${ }^{[42,43]}$ In principle, the metal films could be grown epitaxially on metal oxides and, if the metal oxide substrates are prepared with chiral terrace-step-kink structures, the metal film may grow enantiomorphically and retain the chirality of the substrate. Initial work on this problem has suggested that enantiomorphic heteroepitaxial growth of metals on naturally chiral oxide surfaces can be achieved and may serve as a route to inexpensive naturally chiral metal surfaces.

\section{Adsorbate-induced formation of naturally chiral surfaces}

An adsorbed chiral molecule imparts chirality to a surface merely by its presence. However, it is possible that adsorbate-induced reconstruction of achiral metal surfaces can yield homochiral high Miller index facets with nanoscale kink structures that are naturally chiral. This method of inducing chirality in a surface is distinct from chiral templating in the sense that the chirality of the substrate could be retained even if the adsorbate were removed. This can be thought of as chiral imprinting. Imprinting could serve as a potential route to the inexpensive production of high area chiral surfaces. An example of chiral imprinting arises during amino acid adsorption on the otherwise achiral $\mathrm{Cu}(100)$ surface. ${ }^{[58-60]}$ Step bunching on the $\mathrm{Cu}(100)$ surface in the presence of adsorbed L-lysine causes the formation of a homochiral set of $\{3,1,17\}$ facets. The chirality of the facets is dictated by the chirality of the L-lysine. Although achiral amino acids will induce the formation of $\{3,1,17\}$ facets on the $\mathrm{Cu}(100)$ surface, they are present in both $R$ and $S$ forms.

\section{TECHNOLOGICAL IMPACT OF CHIRAL SURFACES}

The potential technological impact of chiral surfaces is enormous because they can, in principle, play an important role in the processing of chiral pharmaceuticals worth an estimated US\$100 billion per year. At this point in time, the most highly developed application is as stationary phases for chiral chromatography. Chiral columns are often formed by templating of materials such as silica with enantiomerically pure chiral ligands. ${ }^{[9,10]}$ Templating 
has also been shown to produce enantioselective heterogeneous catalysts, which, in principle, offer some advantages over homogeneous catalysts during scaleup. Thus chiral templating of surfaces is a demonstrated technology with growing opportunities for application in pharmaceuticals production.

The study of the enantioselective surface chemistry of naturally chiral surfaces and, in particular, those derived from kinked high Miller index metal surfaces is in its infancy. In principle, they offer potential advantages over templated surfaces in that they may be more thermally and chemically robust because they do not rely on organic ligands as the source of chirality. Another opportunistic niche that the naturally chiral surfaces may fill is in the development of microscale reactors. In reactors based on single-crystalline $\mathrm{Si}$, it is easy to imagine that one could make channels that expose naturally chiral surfaces, and that such an element in a microreactor might then be used for enantioselective chemical processes. Although the impact of naturally chiral surfaces is yet to be determined, there are a number of exciting opportunities. Furthermore, the possible role that naturally chiral surfaces may have played in dictating the origins of the homochirality of life on Earth makes their study intrinsically intriguing.

\section{CONCLUSION}

Of the three types of chiral surfaces described in this article, the most well developed are those that are produced by templating with chiral organic modifiers. Although the mechanisms and adsorbate/substrate interactions that impart enantioselectivity on these surfaces are not understood in great detail, some such surfaces have been highly optimized to give highly enantioselective separations and catalytic reactions. Naturally chiral surfaces can be generated from materials that are chiral in bulk, or achiral materials cleaved to expose surface structures that are chiral at the nanoscale. The crystalline nature of these structures makes them good candidates for a detailed study of the origins of enantiospecificity on their surfaces. Furthermore, they have demonstrated high enantioselectivity for both reactions and separations. The limitation in their practical use is the need for methods of preparation that yield naturally chiral surfaces in high area form. Although possible routes exist, these have yet to be explored.

\section{ACKNOWLEDGMENTS}

We would like to thank Prof. David Sholl and Prof. Greg Rohrer for their helpful discussions. The authors' work on naturally chiral crystals is supported by the NSF through grant no. CTS-0216170 and by the DOE through grant no. DMR-0079996.

\section{REFERENCES}

1. In Chiral Reactions in Heterogeneous Catalysis, Proceedings of the First European Symposium on Chiral Reactions in Heterogeneous Catalysis, Brussels, Belgium, October 25-26; Jannes, G., Dubois, V., Eds.; Plenum Publishers, 1993; 5.

2. Jones, M. Organic Chemistry; W. W. Norton \& Company: New York, 1997.

3. International Tables for Crystallography, 2nd Ed.; Hahn, T., Ed.; Space Group Symmetry, Kluwer Academic Publishers: Dordrecht, 1989; 4.

4. Federsel, H.J. Drug chirality-Scale-up, manufacturing, and control. Chemtech 1993, 23 (12), 24-33.

5. Stinson, S.C. Chiral drugs. Chem. Eng. News 1994, 19, 38.

6. Nugent, W.A.; Rajan Babu, T.V.; Burk, M.J. Beyond nature's chiral pool: Enantioselective catalysis in industry. Science (Washington, DC) 1993, 259 (5094), 479-483.

7. Smith, G.V.; Notheisz, F. Heterogeneous Catalysis in Organic Chemistry; Academic Press: San Diego, 1999.

8. Webb, G.; Wells, P.B. Asymmetric hydrogenation. Catal. Today 1992, 12 (2-3), 319-337.

9. Pirkle, W.H.; Pochapsky, T.C. Considerations of chiral recognition relevant to the liquid chromatography separation of enantiomers. Chem. Rev. (Washington, DC) 1989, 89 (2), 347-362.

10. Francotte, E.R. Enantioselective chromatography as a powerful alternative for the preparation of drug enantiomers. J. Chromatogr., A 2001, 906 (1-2), 379-397.

11. Izumi, Y. Modified Raney nickel (MRNi) catalyst: Heterogeneous enantiodifferentiating (asymmetric) catalyst. Adv. Catal. 1983, 32, 215-271.

12. Hoek, A.; Sachtler, W.M.H. Enantioselectivity of nickel catalysts modified with tartaric acid or nickel tartrate complexes. J. Catal. 1979, 58 (2), 276-286.

13. Hoek, A.; Woerde, H.M.; Sachtler, W.M.H. Enantioselectivity of nickel catalysts modified with tartaric acid or nickel tartrate complexes. Stud. Surf. Sci. Catal. 1981, 7, 376-389.

14. Keane, M.A. Adsorption of optically pure alanine on silica-supported nickel and the consequent catalytic enantioselectivity. Langmuir 1994, 10 (12), 4560-5.

15. Fu, L.; Kung, H.H.; Sachtler, W.M.H. Particle size effect on enantioselective hydrogenation of methyl 
acetoacetate over silica-supported nickel catalyst. J. Mol. Catal. 1987, 42 (1), 29-36.

16. Nitta, Y.; Imanaka, T.; Teranishi, S. Preparation chemistry of precipitated nickel-silica catalysts for enantioselective hydrogenation. J. Catal. 1985, 96 (2), 429-438.

17. Nitta, Y.; Utsumi, T.; Imanaka, T.; Teranishi, S. Effect of preparation variables on morphological and catalytic properties of precipitated nickel-silica catalysts. Chem. Lett. 1984, 8, 1339-1342.

18. Harada, T.; Izumi, Y. Improved modified Raney nickel catalyst for enantioface-differentiating (asymmetric) hydrogenation of methyl acetoacetate. Chem. Lett. 1978, 11, 1195-1196.

19. Bostelaar, L.J.; Sachtler, W.M.H. The role of alkali halides in the enantioselective hydrogenation of a prochiral keto compound over modified nickel catalysts. J. Mol. Catal. 1984, 27 (3), 387-395.

20. Orito, Y.; Imai, S.; Niwa, S.; Nguyen Gia, H. Asymmetric hydrogenation of methyl benzoylformate using platinum-carbon catalysts modified with cinchonidine. Yuki Gosei Kagaku Kyokaisho 1979, 37 (2), 173-174.

21. Sutherland, I.M.; Ibbotson, A.; Moyes, R.B.; Wells, P.B. Enantioselective hydrogenation: I. Surface conditions during methyl pyruvate hydrogenation catalyzed by cinchonidine-modified platinum/silica (EUROPT-1). J. Catal. 1990, 125 (1), 77-88.

22. Meheux, P.A. University of Hull, 1991.

23. Blaser, H.U.; Jalett, H.P.; Wiehl, J. Enantioselective hydrogenation of alpha-keto esters with cinchonamodified platinum catalysts: Effect of acidic and basic solvents and additives. J. Mol. Catal. 1991, 68 (2), 215-222.

24. Tungler, A.; Mathe, T.; Tarnai, T.; Fodor, K.; Toth, G.; Kajtar, J.; Kolossvary, I.; Herenyi, B.; Sheldon, R.A. (-)-Dihydroapovincaminic acid ethyl ester, preparation and use as a chiral modifier in enantioselective heterogeneous catalytic hydrogenations. Tetrahedron: Asymmetry 1995, 6 (9), 23952402.

25. Blaser, H.U. The chiral pool as a source of enantioselective catalysts and auxiliaries. Chem. Rev. (Washington, DC) 1992, 92 (5), 935-952.

26. Baiker, A. Progress in asymmetric heterogeneous catalysis: Design of novel chirally modified platinum metal catalysts. J. Mol. Catal., A Chem. 1997, 115 (3), 473-493.

27. Schurch, M.; Heinz, T.; Aeschimann, R.; Mallat, T.; Pfaltz, A.; Baiker, A. Design of new modifiers for the enantioselective hydrogenation of ethyl pyruvate. J. Catal. 1998, 173 (1), 187-195.

28. Barlow, S.M.; Kitching, K.J.; Haq, S.; Richardson, N.V. A study of glycine adsorption on a $\mathrm{Cu}\{110\}$ surface using reflection absorption infrared spectroscopy. Surf. Sci. 1998, 401 (3), 322-335.

29. Booth, N.A.; Woodruff, D.P.; Schaff, O.; Giessel, T.; Lindsay, R.; Baumgartel, P.; Bradshaw, A.M. Determination of the local structure of glycine adsorbed on $\mathrm{Cu}(110)$. Surf. Sci. 1998, 397 (1-3), 258-269.

30. Nyberg, M.; Hasselstrom, J.; Karis, O.; Wassdahl, N.; Weinelt, M.; Nilsson, A.; Pettersson, L.G.M. The electronic structure and surface chemistry of glycine adsorbed on $\mathrm{Cu}(110)$. J. Chem. Phys. 2000, 112 (12), 5420-5427.

31. Williams, J.; Haq, S.; Raval, R. The bonding and orientation of the amino acid L-alanine on $\mathrm{Cu}\{110\}$ determined by RAIRS. Surf. Sci. 1996, 368 (1-3), 303-309.

32. Raval, R.; Baddeley, C.J.; Haq, S.; Louafi, S.; Murray, P.; Muryn, C.; Lorenzo, M.O.; Williams, J. Complexities and dynamics of the enantioselective active site in heterogeneous catalysis. Stud. Surf. Sci. Catal. 1999, 122, 11-22. (Reaction Kinetics Development of Catalytic Processes).

33. Lahav, N. Biogenesis: Theories of Life's Origins; Oxford University Press: New York, 1999.

34. Tsuchida, R.; Kobayashi, M.; Nakamura, A. Asymmetric adsorption of complex salts on quartz. J. Chem. Soc. Jpn. 1935, 56, 1339-1345.

35. Karagounis, G.; Coumonlos, G. A new method for resolving a racemic compound. Nature 1938, 142, $162-163$.

36. Bonner, W.A.; Kavasmaneck, P.R.; Martin, F.S.; Flores, J.J. Asymmetric adsorption of alanine by quartz. Science 1974, 186 (4159), 143-144.

37. Bonner, W.A.; Kavasmaneck, P.R.; Martin, F.S.; Flores, J.J. Asymmetric adsorption by quartz. Model for the prebiotic origin of optical activity. Orig. Life 1975, 6 (3), 367-376.

38. Soai, K.; Sato, I.; Shibata, T. Asymmetric autocatalysis and the origin of chiral homogeneity in organic compounds. Chem. Rec. 2001, 1 (4), 321-332.

39. Cody, A.M.; Cody, R.D. Chiral habit modifications of gypsum from epitaxial-like adsorption of stereospecific growth inhibitors. J. Cryst. Growth 1991, 113 (3-4), 508-519.

40. Orme, C.A.; Noy, A.; Wierzbicki, A.; McBride, M.T.; Grantham, M.; Teng, H.H.; Dove, P.M.; DeYoreo, J.J. Formation of chiral morphologies through selective binding of amino acids to calcite surface steps. Nature (London) 2001, 411 (6839), 775-779.

41. Hazen, R.M.; Filley, T.R.; Goodfriend, G.A. Selective adsorption of L- and D-amino acids on calcite: Implications for biochemical homochirality. Proc. Natl. Acad. Sci. U. S. A. 2001, 98 (10), 5487-5490. 
42. Terent'ev, A.P.; Klabunovskii, E.I.; Patrikeev, V.V. Asymmetric synthesis with the aid of catalysts deposited on right and left quartz. Dokl. Akad. Nauk Souza Sov. Social. Resp., A 1950, 74, 947-950.

43. Klabunovskii, E.I.; Patrikeev, V.V. Mechanism of the asymmetrizing effect of metal catalysts deposited on right and left quartz. Dokl. Akad. Nauk Souza Sov. Social. Resp., A 1951, 78, 485-487.

44. Ahmadi, A.; Attard, G.; Feliu, J.; Rodes, A. Surface reactivity at "chiral" platinum surfaces. Langmuir 1999, 15 (7), 2420-2424.

45. Sholl, D.S.; Asthagiri, A.; Power, T.D. Naturally chiral metal surfaces as enantiospecific adsorbents. J. Phys. Chem., B 2001, 105 (21), 4771-4782.

46. Asthagiri, A.; Feibelman, P.J.; Sholl, D.S. Thermal fluctuations in the structure of naturally chiral $\mathrm{Pt}$ surfaces. Top. Catal. 2002, 18 (3-4), 193-200.

47. Power, T.D.; Asthagiri, A.; Sholl, D.S. Atomically detailed models of the effect of thermal roughening on the enantiospecificity of naturally chiral platinum surfaces. Langmuir 2002, 18 (9), 3737-3748.

48. Dieluweit, S.; Ibach, H.; Geisen, M.; Einstein, T.L. Orientation dependence of step stiffness: Failure of SOS and ising models to describe experimental data. Phys. Rev. Lett., in press.

49. McFadden, C.F.; Cremer, P.S.; Gellman, A.J. Adsorption of chiral alcohols on "chiral" metal surfaces. Langmuir 1996, 12 (10), 2483-2487.

50. Sholl, D.S. Adsorption of chiral hydrocarbons on chiral platinum surfaces. Langmuir 1998, 14 (4), $862-867$.

51. Horvath, J.D.; Gellman, A.J. Enantiospecific desorption of $R$ - and $S$-propylene oxide from a chiral $\mathrm{Cu}(643)$ surface. J. Am. Chem. Soc. 2001, 123 (32), $7953-7954$.
52. Horvath, J.D.; Gellman, A.J. Enantiospecific desorption of chiral compounds from chiral $\mathrm{Cu}(643)$ and achiral $\mathrm{Cu}(111)$ surfaces. J. Am. Chem. Soc. 2002, 124 (10), 2384-2392.

53. Horvath, J.; Kamakoti, P.; Sholl, D.S.; Gellman, A.J. Enantioselective separation on a naturally chiral surface. Nature, in press.

54. Attard, G.A.; Ahmadi, A.; Feliu, J.; Rodes, A.; Herrero, E.; Blais, S.; Jerkiewicz, G. Temperature effects in the enantiomeric electro-oxidation of Dand L-glucose on Pt\{643\}S. J. Phys. Chem., B 1999, 103 (9), $1381-1385$.

55. Attard, G.A. Electrochemical studies of enantioselectivity at chiral metal surfaces. J. Phys. Chem., B 2001, 105 (16), 3158-3167.

56. Attard, G.A.; Harris, C.; Herrero, E.; Feliu, J. The influence of anions and kink structure on the enantioselective electro-oxidation of glucose. Faraday Discuss. 2002, 121, 253-266. (Dynamic Electrode Surface).

57. Asthagiri, A.; Sholl, D.S. First principles study of $\mathrm{Pt}$ adhesion and growth on SrO- and $\mathrm{TiO}_{2}$ terminated $\mathrm{SrTiO}_{3}(100)$. J. Chem. Phys. 2002, 116 (22), 9914-9925.

58. Zhao, X.; Gai, Z.; Zhao, R.G.; Yang, W.S.; Sakurai, T. Adsorption of glycine on $\mathrm{Cu}(001)$ and related step faceting and bunching. Surf. Sci. 1999, 424 (23), L347-L351.

59. Zhao, X.; Zhao, R.G.; Yang, W.S. Adsorption of alanine on $\mathrm{Cu}(001)$ studied by scanning tunneling microscopy. Surf. Sci. 1999, 442 (2), L995-L1000.

60. Zhao, X.; Zhao, R.G.; Yang, W.S. Scanning tunneling microscopy investigation of L-lysine adsorbed on $\mathrm{Cu}(001)$. Langmuir 2000, 16 (25), 9812-9818. 


\section{Request Permission or Order Reprints Instantly!}

Interested in copying and sharing this article? In most cases, U.S. Copyright Law requires that you get permission from the article's rightsholder before using copyrighted content.

All information and materials found in this article, including but not limited to text, trademarks, patents, logos, graphics and images (the "Materials"), are the copyrighted works and other forms of intellectual property of Marcel Dekker, Inc., or its licensors. All rights not expressly granted are reserved.

Get permission to lawfully reproduce and distribute the Materials or order reprints quickly and painlessly. Simply click on the "Request Permission/ Order Reprints" link below and follow the instructions. Visit the U.S. Copyright Office for information on Fair Use limitations of U.S. copyright law. Please refer to The Association of American Publishers' (AAP) website for guidelines on Fair Use in the Classroom.

The Materials are for your personal use only and cannot be reformatted, reposted, resold or distributed by electronic means or otherwise without permission from Marcel Dekker, Inc. Marcel Dekker, Inc. grants you the limited right to display the Materials only on your personal computer or personal wireless device, and to copy and download single copies of such Materials provided that any copyright, trademark or other notice appearing on such Materials is also retained by, displayed, copied or downloaded as part of the Materials and is not removed or obscured, and provided you do not edit, modify, alter or enhance the Materials. Please refer to our Website User Agreement for more details.

\section{Request Permission/Order Reprints}

Reprints of this article can also be ordered at http://www.dekker.com/servlet/product/DOI/101081EENN120013840 